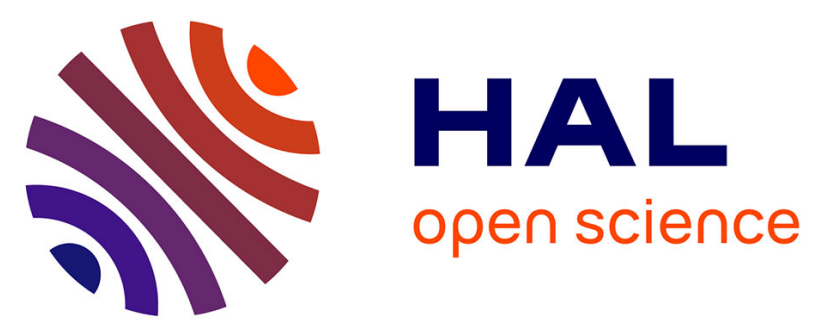

\title{
Etude exploratoire des relations entre conscience émotionnelle, partage social des émotions, états anxieux et états dépressifs
}

Aurélie Pasquier, Jean-Louis Pedinielli

\section{- To cite this version:}

Aurélie Pasquier, Jean-Louis Pedinielli. Etude exploratoire des relations entre conscience émotionnelle, partage social des émotions, états anxieux et états dépressifs. L'Encéphale, 2010, Dépression, 36 (S2), pp.97-104. 10.1016/j.encep.2009.01.007 . hal-01271923

\section{HAL Id: hal-01271923 \\ https://hal-amu.archives-ouvertes.fr/hal-01271923}

Submitted on 9 Feb 2016

HAL is a multi-disciplinary open access archive for the deposit and dissemination of scientific research documents, whether they are published or not. The documents may come from teaching and research institutions in France or abroad, or from public or private research centers.
L'archive ouverte pluridisciplinaire $\mathbf{H A L}$, est destinée au dépôt et à la diffusion de documents scientifiques de niveau recherche, publiés ou non, émanant des établissements d'enseignement et de recherche français ou étrangers, des laboratoires publics ou privés. 
Etude exploratoire des relations entre conscience émotionnelle, partage social des émotions, états anxieux et états dépressifs

Exploratory study of relations between emotional awareness, social sharing of emotions, anxious and depression states

Aurélie Pasquier (1), Jean-Louis Pedinielli (2)

1 Maître de conférence en psychopathologie*

2 Professeur de psychopathologie*

*Centre de recherches en Psychologie de la Connaissance, du Langage et de l'Emotion (PsyCLE, EA3273). Axe thématique III «Emotions, Construction du réel et Pathologie ». Aix-Marseille Université

21, avenue Robert Schuman

13621 Aix-en-Provence Cedex 1

Mail : aureliepasquier@free.fr

Article publié dans la revue L'Encéphale en juin 2010 (vol. 36, S2, p.97-104) 
Résumé :

Les états anxieux et dépressifs sont de plus en plus répandus. Leur prévalence respective actuelle est estimée autour des $12 \%$. Cette recherche vise à étudier le fonctionnement émotionnel spécifique aux individus pouvant présenter chaque type d'état (anxieux et/ou dépressif). Notre objectif est donc d'analyser les relations pouvant exister entre les dimensions d'anxiété et de dépression, le niveau de conscience émotionnelle (capacités d'identification et de différenciation de ses propres émotions et de celles d'autrui) et le processus de partage social des émotions (mécanisme de régulation émotionnelle interpersonnelle).

L'échantillon de notre étude compte 107 participants volontaires issus de cabinets de médecine générale d'une part et de la population tout-venant d'autre part. L'âge moyen de l'ensemble des sujets est de 43,21 ans $( \pm 12,76)$ avec une proportion de 13 hommes pour 94 femmes. Deux groupes de sujets ont été constitués sur la base de leurs niveaux d'anxiété et de dépression évalués par 1'HAD (Hospital Anxiety and Depression Scale ; Zigmond et coll., 1983). Le groupe «AD» est constitué de sujets anxieux et anxio-dépressifs $(N=60)$. Le groupe «NAD » est constitué de sujets non anxieux et non dépressifs $(\mathrm{N}=47)$. Des échelles auto-évaluatives composent notre protocole : l'échelle d'évaluation des niveaux de conscience émotionnelle (LEAS ; Lane et coll., 1990) et l'échelle d'évaluation du partage social des émotions (Rimé, 1991) utilisée sur la base du rappel d'un événement négatif marquant.

Les analyses corrélationnelles montrent la présence d'une relation négative entre le niveau de conscience émotionnelle et la dimension d'anxiété $(\mathrm{r}=-.26 ; \mathrm{p}=.04)$, mais positive entre le niveau conscience et celui de dépression ( $\mathrm{r}=.37 ; \mathrm{p}=.003)$. De plus, les individus anxieux manifesteraient une tendance à l'inhibition du partage social des émotions $(\mathrm{r}=.26$; $\mathrm{p}=.05$ ) dans le but de ne pas réactiver le vécu émotionnel négatif, alors que dans le cas de la dépression, il s'agit de l'inhibition de certains aspects du vécu émotionnel ( $\mathrm{r}=.33 ; \mathrm{p}=.01)$ que l'individu ne souhaite pas soumettre au regard d'autrui.

Les résultats apportent des données intéressantes concernant une population «infraclinique » (sujets anxio-dépressifs pris en charge en cabinets de généraliste). Leur portée est cependant limitée par la taille de l'échantillon qui pourrait être élargi pour poursuivre nos investigations. Cette étude permet d'approfondir les connaissances sur les capacités d'identification et de régulation des émotions d'individus présentant des symptômes anxieux et/ou dépressifs.

Mots-clés : Anxiété, dépression, conscience émotionnelle, partage social des émotions. 
Summary:

The anxious and depressive states are increasingly common. Their respective current prevalence is estimated around $12 \%$. This research aims to study how the emotional specific individuals may submit each type of condition (anxiety and/or depression). Our objective is to analyse the relationships that might exist between these states, the level of emotional awareness (capacity for the identification and differentiation of one's own emotions and those of others) and the social sharing of emotions process (mechanism interpersonal emotional regulation).

The sample is composed by 107 volunteers from general medical practice on the one hand and population-run second. The average age of all subjects is 43.21 years $( \pm 12.76)$ with a ratio of 13 men for 94 women. Two groups of subjects were formed on the basis of their levels of anxiety and depression assessed by the HAD (Hospital Anxiety and Depression Scale; Zigmond and al., 1983). The «AD » group consists of subjects anxious and anxietydepression $(\mathrm{N}=60)$. The « NAD » group consists of subjects not anxious and not depressive $(\mathrm{N}=47)$. Scales of self-evaluation up our protocol: the assessment of levels of emotional awareness (LEAS; Lane and al., 1990) and the assessment of social sharing emotions (Rimé, 1991) used on the basis of the recall of a significant negative event.

Correlational analysis showed the presence of a negative relationship between the level of emotional awareness and dimension of anxiety $(\mathrm{r}=-.26, \mathrm{p}=.04)$, but positive between the level of awareness and depression ( $\mathrm{r}=.37 ; \mathrm{p}=.003)$. In addition, individuals anxious to demonstrate a trend of social inhibition sharing emotions $(\mathrm{r}=-.26 ; \mathrm{p}=.05)$ in order not to reactivate the negative emotional experience, whereas in the case of depression, is the inhibition of certain aspects emotional experiences $(r=.33 ; p=.01)$ that the individual does not wish to submit to the other's view.

The results show interesting data on a population "sub-clinical" (anxiety-depressive subjects supported in general medicine). Their scope is limited by the size of the sample that could be expanded to continue our investigations. This study helps to deepen knowledge about the capabilities of identification and regulation of emotions of people showing symptoms of anxiety and/or depression.

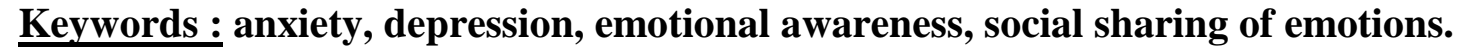




\section{Introduction}

Les symptomatologies anxieuse et dépressive constituent de réels problèmes de santé publique. Selon l'Organisation Mondiale de la Santé, chacun de ces deux ensembles symptomatologiques, souvent co-morbides, toucherait environ $12 \%$ de la population générale.

La population la plus à risque serait surtout féminine ( 2 femmes pour un homme), de tous âges et de tous milieux sociaux (même si les milieux socio-économiques défavorisés seraient surreprésentés).

Cependant, les études épidémiologiques montrent que concrètement les individus souffrant d'anxiété et/ou de dépression n'entament généralement pas de démarches visant à consulter un thérapeute.

Plusieurs études font apparaître que plus de la moitié des individus anxieux comme des dépressifs consultent exclusivement leur médecin généraliste $[11,12,28]$. Lorsqu'il existe chez ces patients une prise en charge médicamenteuse, elle aurait tendance à se prolonger dans le temps malgré les recommandations faites pour ces types de traitement (anxiolytiques, antidépresseurs).

D'un point de vue psychopathologique, l'anxiété comme la dépression seraient associées à des difficultés d'identification et de verbalisation des émotions propres $[1,3,9,10]$ plus généralement regroupées sous le terme d'alexithymie [22,29].

Ce concept qui signifie littéralement l'absence de mots pour décrire ses émotions entretiendrait des relations positives significatives avec la présence d'états anxieux et/ou dépressifs. Ceci pourrait alors expliquer les trajectoires de prise en charge empruntées par des individus souffrant de tels symptômes. En effet, si les symptomatologies anxieuse et dépressive sont associées à des difficultés de reconnaissance et de verbalisation des émotions alors les individus qui présentent cet(s) état(s) auront sans doute du mal à démêler ce qui est de l'ordre du physiologique et du psychologique.

Cependant, une dimension sociale interviendrait selon nous et pourrait constituer un facteur explicatif important du refus de l'individu anxieux et/ou dépressif au suivi, quel qu'il soit. En effet, la stigmatisation des termes de dépression et d'anxiété participerait aux réticences que peuvent manifester ces individus à faire état de leur trouble. Plusieurs travaux montrent que le vécu même de la maladie dépressive et/ou anxieuse (tension interne, culpabilité, honte,...) freinerait toute demande de soin $[18,19]$.

Fort de ces différents constats, nous avons dès lors choisi d'appréhender les composantes $\mathrm{du}$ fonctionnement émotionnel (notamment le niveau de conscience 
émotionnelle) d'individus souffrant d'anxiété et/ou de dépression en y introduisant l'évaluation d'une composante sociale (processus de partage social des émotions) [25].

\subsection{La conscience émotionnelle}

La conscience émotionnelle décrit la capacité d'un individu à faire l'expérience d'états subjectifs différenciés, à les interpréter correctement ainsi qu'à les imaginer et les identifier chez les autres. Selon le modèle de Lane et Schwartz, elle serait un type de traitement cognitif correspondant à cinq niveaux de transformation structurale tout au long du développement [14]. Par une définition en négatif de ce concept, on retrouve des éléments qui rappellent ceux servant à décrire l'alexithymie. Des difficultés de différenciation et de description des états émotionnels sont non sans rappeler les caractéristiques propres à la dimension d'alexithymie [29]. C'est pour cela que Taylor et collaborateurs proposent de transposer le modèle de Lane et Schwartz à cette dimension [31]. Dans cette perspective, un score élevé d'alexithymie correspondrait aux stades initiaux du développement émotionnel. A l'opposé, le dernier stade rend compte du fonctionnement d'individus dépourvus de caractéristiques alexithymiques. Ainsi, Lane et collaborateurs ont émis l'hypothèse que les fonctionnements émotionnels pathologiques rencontrés tels que l'alexithymie pourraient correspondre à des arrêts développementaux à des stades précoces du développement émotionnel [15].

Des travaux ont cherché à mettre en évidence les relations qui existent entre des niveaux de conscience émotionnelle et les symptomatologies anxieuse et dépressive. L'appréhension de cette dimension peut fournir des éléments de compréhension concernant ces symptomatologies dans la mesure où elles seraient associées à un faible niveau de développement de la conscience émotionnelle [4,7]. De plus on connaît les liens qu'entretiennent ces symptomatologies avec l'alexithymie [17].

\subsection{Le partage social des émotions}

Rimé $[25,26]$ a proposé l'expression de «partage social des émotions » (PSE) pour rendre compte de la propension des individus à communiquer avec leur environnement suite à des événements émotionnels. Si les premières observations de ce phénomène sont issues de l'étude des traumatismes émotionnels (catastrophes naturelles, guerres, etc.), un certain nombre de travaux a mis en évidence que le partage social des émotions concerne l'ensemble des événements émotionnels. Les données suggèrent qu'il constitue une conséquence typique 
de l'émotion voire même une facette de l'expérience émotionnelle. Partager socialement ses émotions permettrait de réguler ses éprouvés subjectifs [27].

Habituellement, ce n'est pas un travail cognitif de mise en sens de l'événement vécu qui est visé par le partage social des émotions. Les bénéfices sont plus d'ordre sociaux et émotionnels. En effet, des travaux montrent que lorsque l'individu partage ses émotions, les phénomènes physiologiques et émotionnels consécutifs à l'expérience émotionnelle sont améliorés. De plus, un rapprochement affectif se produit, renforçant les liens sociaux de l'individu. A l'inverse, l'inhibition du partage social des émotions entraînerait des coûts sociaux et émotionnels pour les deux partenaires d'une conversation émotionnelle [5].

Au cours de notre développement nous avons appris à identifier, nommer et verbaliser nos émotions. Ces différents moyens développés et mis en œuvre aident à la régulation des éprouvés subjectifs. Il existerait de fait deux niveaux de régulation émotionnelle: un processus interne par la reconnaissance et l'indentification des émotions (conscience émotionnelle), et un processus interpersonnel de régulation, par le partage social des émotions.

\subsection{Objectifs}

A travers cette étude, nous avions plusieurs objectifs exploratoires : le premier était de déterminer les différentes composantes du fonctionnement émotionnel global et notamment celle de régulation émotionnelle interpersonnelle (partage social des émotions) spécifique aux états anxieux et aux états dépressifs dans un échantillon de patients consultant en cabinet de médecine générale. Le deuxième objectif était d'analyser ces différentes relations afin de proposer un modèle exploratoire de relation spécifique à chaque état (anxieux et dépressif). En effet, si dans un premier temps nous nous attendons à constater l'existence d'une corrélation positive entre états anxieux et dépressifs, nous considérons dans un second temps que ces derniers possèdent chacun un fonctionnement émotionnel spécifique.

\section{Méthodologie}

\subsection{Echantillon}

Notre échantillon est constitué de 107 sujets (H/F). Ils ont tous été recrutés sur la base du volontariat de deux manières différentes. Une partie des sujets sont des patients de cabinets 
de médecine générale de la région PACA (Aix-en-Provence, Marseille, Toulon). Ils ont accepté de participer à l'étude après avoir pris connaissance du formulaire de présentation de la recherche et de consentement éclairé. La période de recrutement s'est étalée sur sept mois et le taux de refus a été environ de 50\% (la part des hommes dans la proportion de refus était de l'ordre de $70 \%$ par rapport aux femmes). Les protocoles étaient mis à disposition par les médecins eux-mêmes dans leurs cabinets. Ils étaient destinés à des patients préalablement repérés par le médecin comme présentant des symptômes anxieux et/ou dépressifs (utilisation de la liste des critères servant à décrire l'anxiété généralisée et l'épisode dépressif majeur, DSM-IV-TR). La proposition faite aux patients, de remplir le protocole de recherche, se faisait au grès des rendez-vous pris par les patients eux-mêmes. La possibilité de joindre l'investigatrice de la recherche (une psychologue) était proposée sur le formulaire, notamment pour obtenir par la suite des informations sur les résultats globaux de la recherche. Dans tous les cas, le médecin proposait une orientation psychologique (avec un professionnel autre que l'investigatrice de la recherche) à l'issue de l'entretien, suite à la consultation.

L'autre partie des sujets a été recrutée dans différentes institutions (banques, écoles, universités, entreprises). Plusieurs situations professionnelles sont représentées (étudiants, secrétaires, professeurs des écoles, cadres supérieurs, etc.). Les protocoles accompagnés du formulaire de présentation de la recherche et de consentement éclairé étaient mis à disposition dans l'institution pour les personnes volontaires.

L'âge moyen des sujets est de 43,21 ( $\pm 12,76)$ avec un sexe ratio (H/F) de 13/94. Nous avons constitué deux groupes homogènes non appariés de sujets selon leur niveau d'anxiété et leur niveau de dépression. Tous les sujets de cette étude présentaient chacun un score d'anxiété et un autre de dépression quel que soit leur groupe d'appartenance.

Un premier groupe $(\mathrm{N}=60$ avec un sexe ratio $\mathrm{H} / \mathrm{F}$ de 7/53) est composé des sujets issus des cabinets de médecine générale. Leur score d'anxiété atteint la note seuil (égale à 8) à partir de laquelle on considère que le niveau de symptômes est pathologique [2]. Parmi ces sujets certains présentent des scores de dépression qui atteignent aussi la valeur seuil (égale à 8) permettant de considérer le caractère pathologique des symptômes (nombre d'anxieux et de dépressifs sur effectif global du groupe : 13/60). Ce groupe composé de sujets anxieux et/ou dépressifs est noté $\mathrm{AD}$.

Un deuxième groupe est constitué de sujets tout-venant dont les scores n'atteignent pas les valeurs seuils permettant de statuer sur le critère pathologique des niveaux d'anxiété et de dépression. Ce groupe composé de sujets non anxieux et non dépressifs est noté NAD. 
Constituer ces deux groupes nous a essentiellement permis de définir notre ensemble de sujets fonctionnant à un niveau dit pathologique (groupe $\mathrm{AD}$ ). Cependant, ces derniers n'ayant pas été recrutés dans des lieux de prise en charge des pathologies anxieuse et dépressive, la majorité d'entre eux présentait des niveaux modérés d'anxiété et/ou de dépression (cf. tableau 1). Il s'agit d'une population que l'on pourrait qualifier d'infraclinique puisque nous sommes loin des niveaux de pathologie majeure habituellement étudiés. Malgré tout, une souffrance ressentie existe et il est ici question de son exploration.

\subsection{Les instruments}

Mesures des niveaux d'anxiété et de dépression

Le «Hospital Anxiety Depression » (HAD) est un auto-questionnaire structuré en 14 items. Il évalue le niveau actuel de la symptomatologie anxieuse et dépressive [32]. Cet outil a été validé en Français [16]. Il nous semble intéressant pour notre étude puisque la version française du HAD a fait l'objet de travaux réalisés chez des sujets consultant en médecine générale. Le participant doit répondre rapidement à chacune des affirmations en faisant correspondre le chiffre qui convient le mieux à son état actuel. L'utilisation de cette échelle assez courte est souvent bien acceptée. Un score est attribué à chaque répondant en anxiété et en dépression en fonction des réponses choisies. Nous nous sommes référés à la cotation de Barczack et collaborateurs [2] selon laquelle une note au moins égale à 8 constituerait le seuil optimal pour considérer le caractère pathologique des symptômes avec des sensibilités respectives de 82 et $70 \%$ et des spécificités de 94 et $68 \%$.

\section{Mesure du niveau de conscience émotionnelle}

Nous avons utilisé l'échelle de niveaux de conscience émotionnelle («Level of Emotional Awareness Scale », LEAS) développée par Lane et collaborateurs [13]. Il s'agit d'un auto-questionnaire composé de 20 situations dans lesquelles on demande aux sujets de se représenter dans une courte scène le mettant en interaction avec une autre personne. Les réponses sont cotées de 0 à 5 et permettent d'obtenir un score global de 0 à 100 avec deux sous-scores évaluant les niveaux de conscience émotionnelle intra- et inter-subjectif. L'ensemble des cotations pour cette échelle ont été réalisées par une seule et même personne pour éviter des écarts dans l'appréciation des réponses. La structure factorielle de l'adaptation française de l'échelle ainsi que ses qualités psychométriques sont satisfaisantes [6].

Mesure du partage social des émotions 
Il n'y a pas à proprement parler d'échelle de Partage Social des Emotions (PSE). Dans les recherches menées par Bernard Rimé, sont utilisés des moyens d'évaluer le PSE, et ces moyens varient en fonction de l'étude, de la procédure et des objectifs visés. Quatre procédures existent : la méthode du rappel autobiographique ou «procédure rétrospective », mais aussi la «méthode du journal », la « procédure follow-up » et la méthode expérimentale (induction d'émotions en laboratoire) [26]. Nous avons choisi d'utiliser la première procédure, celle rétrospective. Nous avons fait le choix de nous intéresser à un événement du passé, significatif pour les sujets et à la suite duquel ils ont éprouvé ou non le besoin d'en parler. Plusieurs éléments caractéristiques de l'événement cible et du PSE ont été évalués et chacune des dimensions évaluées ont donné lieu à un score original ne pouvant être additionné à aucun autre score [25,26]. Nous avons également fourni en annexe du questionnaire de PSE, une liste d'événements de vie à laquelle le répondant pouvait se référer s'il en éprouvait le besoin. Cette liste est issue de celle proposée dans le questionnaire d'événements de vie d'Amiel-Lebigre. Elle comporte 53 propositions (dont une question ouverte sur un autre événement auquel le sujet pense et qui n'est pas référencé) qui touchent à différents thèmes de la vie : le deuil, les difficulté/réussite professionnelles, la santé, la vie sociale, familiale, conjugale, etc. Nous avons choisi d'utiliser cette échelle en demandant aux sujets de se rappeler un événement émotionnel négatif considéré comme le plus marquant qu'ils aient pu vivre ces dernières années. Ils devaient ensuite répondre à différentes questions se rapportant à cet événement.

\subsection{Procédure et analyses statistiques}

La passation était individuelle et toutes les tâches étaient présentées dans un ordre fixe (LEAS_HAD_PSE) pour écarter les effets d'ordre de présentation des questionnaires selon les participants, et sans limite de temps. Après avoir rempli les protocoles à leur domicile, il était demandé aux sujets de l'envoyer sous pli anonyme à l'investigateur de la recherche.

Les données ont fait l'objet d'un traitement statistique à l'aide du logiciel Statview 5.0. Nous avons procédé à des comparaisons de moyennes ( $\mathrm{t}$ de student) dans le but de montrer l'existence de différences entre nos deux groupes de sujets, avant de procéder à des analyses corrélationnelles univariées ( $\mathrm{r}$ de Bravais-Pearson) dans le groupe AD uniquement, qui nous permettrons de déterminer la présence de relations spécifiques entre états anxieux, états dépressifs, niveau de conscience émotionnelle et processus de partage social des émotions. 


\section{Résultats}

\subsection{Statistiques descriptives}

\subsubsection{Caractéristiques des groupes «anxieux-dépressifs » (AD) et «non anxieux-}

dépressifs » (NAD)

Les sujets anxieux et/ou dépressifs (AD) ont en moyenne un niveau d'anxiété plus élevé comparativement aux sujets non anxio-dépressifs (tableau 1). La différence entre les deux moyennes est significative. Le score moyen des sujets AD atteint la note seuil (note=8) et permet de les considérer comme anxieux.

\section{$\underline{\text { Tableau } 1}$}

Le niveau de dépression des sujets $\mathrm{AD}$ est en moyenne plus élevé que celui des sujets NAD. La différence entre les deux groupes est significative même si aucun des deux groupes de sujets n'atteignent la note seuil (égale à 8) permettant de considérer le niveau pathologique des symptômes. Le niveau moyen de conscience émotionnelle est inférieur pour le groupe AD quelle que soit la dimension considérée. Les sujets $\mathrm{AD}$ ont donc un niveau de conscience émotionnelle inférieur aux individus tout venant. A l'inverse, il n'existe pas de différences significatives entre les deux groupes pour les composantes du processus de partage social des émotions. Les sujets $\mathrm{AD}$ ne se différencient pas de manière significative des sujets NAD sur ces composantes du PSE.

Par ailleurs, quelque soit les symptômes considérés, le groupe AD est homogène quant à l'âge et le sexe. Il n'existe pas d'effet principal du sexe et de l'âge sur le niveau d'anxiété au sein du groupe $\operatorname{AD}\left(F_{\text {sexe }}(0,68)=0,04 ; p>.05 ; F a ̂ g e(1,81)=0,12 ; p>.05\right)$. Il n'existe pas non plus d'effet de l'âge et du sexe sur le niveau de dépression pour le groupe $\operatorname{AD}\left(F_{\text {sexe }}(12,18)=1,48\right.$; p>.05 et Fâge(1,28)=0,15; p>.05). Il existe par contre un effet de l'âge uniquement sur la dimension de conscience émotionnelle intra-subjective (Fâge $(421,745)=5,955 ; p=.01)$. Il semble que les capacités de différenciation de ses propres états émotionnels soient sous la dépendance de l'âge dans le sens d'une baisse des capacités avec l'âge augmentant. Ces résultats sont en accord avec des données précédemment obtenues où nous avons pu montrer que les sujets les plus âgés tendaient vers une préoccupation diminuante quant à leurs propres états émotionnels [21].

\subsection{Corrélations}




\subsubsection{Etats anxieux, états dépressifs et conscience émotionnelle}

Des analyses corrélationnelles de type Bravais-Pearson entre les états anxieux et dépressifs au sein du groupe AD montrent que le niveau d'anxiété est corrélé positivement de manière significative avec le niveau de dépression (tableau 2). Par conséquent, plus les individus présentent une anxiété état et plus ils manifestent un niveau de symptômes dépressifs important.

\section{$\underline{\text { Tableau } 2}$}

Nous nous sommes ensuite intéressés aux relations pouvant exister entre les niveaux d'anxiété et de conscience émotionnelle, et de la même manière entre ceux de dépression et de conscience émotionnelle. Les analyses corrélationnelles mettent en évidence l'existence de relations inverses avec le niveau de conscience émotionnelle selon l'état du sujet, au sein du groupe $\mathrm{AD}$ (tableau 2). En effet, les niveaux d'anxiété et de conscience émotionnelle entretiennent des relations négatives significatives (relations négatives aussi pour les sousdimensions «conscience émotionnelle intra-subjective » et «conscience émotionnelle intersubjective ») pendant que le niveau de dépression est corrélé positivement de manière significative à celui de conscience émotionnelle (même chose au niveau des sous-dimensions de la conscience émotionnelle). En d'autres termes :

- moins le niveau de conscience émotionnelle est élevé (moins l'individu est capable de se représenter son vécu interne, de le différencier de celui d'autrui) et plus le niveau d'anxiété-état des « sujets AD » est élevé ;

- plus le niveau de conscience émotionnelle est élevé et plus le niveau de dépression des « sujets AD » l'est aussi.

\subsubsection{Etats anxieux, états dépressifs et partage social des émotions}

Les analyses de corrélations univariées mettent en évidence l'existence de relations positives significatives au sein du groupe $\mathrm{AD}$, entre les niveaux d'anxiété et de dépression, et les composantes d'inhibition («inhibition complète du partage social émotionnel», «inhibition d'une partie du vécu émotionnel lors du partage social ») de partage social des émotions :

- plus le niveau d'anxiété est élevé et plus les sujets $\mathrm{AD}$ ont tendance à inhiber le partage social de leurs émotions $(\mathrm{r}=.26 ; \mathrm{p}=.05)$; 
- plus le niveau de dépression est élevé et plus les sujets $\mathrm{AD}$ ont tendance à inhiber une partie seulement du vécu émotionnel lors du partage social de leurs émotions $(r=.33$; $\mathrm{p}=.01)$.

\subsubsection{Conscience émotionnelle et partage social des émotions}

Les analyses corrélationnelles entre ces deux variables et leurs sous-dimensions montrent tout d'abord que la composante d'inhibition du partage social des émotions («s'empêcher de partager ses émotions avec autrui ») ne corrèle pas de manière significative avec le niveau de conscience émotionnelle.

Concernant cette fois les relations pouvant exister entre «l'expression complète du vécu émotionnel » lors du partage social émotionnel et le niveau de conscience émotionnelle, on s'aperçoit que plus les sujets $\mathrm{AD}$ sont conscients des émotions d'autrui (conscience émotionnelle inter-subjective), moins ils expriment de façon complète leurs émotions (ils inhibent une partie de leurs émotions) ( $\mathrm{r}=-.34 ; \mathrm{p}=008)$.

\section{Discussion}

Nos données soulignent que si les niveaux d'anxiété et de dépression sont positivement corrélés entre eux conformément à nos attentes et à la littérature [23], ils ne présentent pas les mêmes types de relations avec le niveau de conscience émotionnelle et le processus de partage social des émotions. Les résultats montrent que l'anxiété-état, au sein d'une population de patients recrutés en médecine générale, est associée à des capacités restreintes de différenciation et de représentation des émotions propres (niveau de conscience émotionnelle). En effet, nous avons pu constater que plus le niveau de conscience émotionnelle était bas et plus le niveau d'anxiété était important. En ce sens, nos résultats sont cohérents avec d'autres déjà obtenus dans la littérature concernant l'existence d'une relation positive entre des difficultés d'identification et de description des émotions et l'anxiété-état [7]. Concernant à présent la symptomatologie dépressive, on a pu observer que celle-ci entretenait une relation positive avec le niveau de conscience émotionnelle. Cela signifie que plus les individus ont une conscience émotionnelle développée (plus ils sont capables de différencier et décrire leurs propres émotions) et plus ils présentent un niveau élevé de dépression. Cette relation intéressante va à l'encontre de certaines études qui montrent que la dépression est davantage liée à des capacités restreintes d'identification et de description des 
émotions [3,4]. Une partie de ces résultats a été obtenue sur la base d'évaluation de dimensions d'alexithymie (difficultés à identifier et décrire ses émotions) par la TAS (Toronto Alexithymia Scale) [3]. Nous considérons que les deux échelles, LEAS et TAS, n'évaluent pas directement la même chose. Des travaux ont cherché à explorer les liens pouvant exister entre ces deux échelles et celle évaluant la honte et la culpabilité ${ }^{1}$. La TAS semble être fortement corrélée avec ces émotions et plus spécifiquement avec la «honte sociale » (honte ressentie à parler de ses émotions à autrui) alors qu'on remarque l'absence de corrélation significative entre la LEAS et ces mêmes émotions [30]. Selon nous, les données obtenues au moyen de la TAS ne peuvent être comparables aux nôtres.

Une autre partie des résultats concernant les relations entre dépression et capacités d'identification et de description des émotions a été obtenue par la LEAS cette fois mais dans une population de sujets manifestant une dépression majeure [4]. Nous postulons que les fonctionnements émotionnels peuvent varier différemment en fonction de l'intensité de la dépression. Nos résultats soulignent que les patients de médecine générale sont en proie à une humeur négative modérée, marquée principalement par la tristesse [24]. Par conséquent, si l'individu est à même de reconnaître ses affects dépressifs, cela signifie qu'il est capable d'utiliser son répertoire émotionnel pour décrire son état émotionnel. Cependant, on pourrait penser que ces capacités d'identification des émotions ne seraient pas suffisantes pour réguler l'humeur de l'individu. En effet, l'individu aurait alors tendance à se focaliser sur ses éprouvés négatifs de sorte qu'il s'en trouverait « débordé » et ne parviendrait pas à les réguler de manière efficace au niveau interne. Ceci nous amène alors à considérer le rôle du processus de partage social des émotions dans sa composante de régulation émotionnelle interpersonnelle.

Les résultats issus de l'analyse des relations entre partage social des émotions et anxiété d'une part, et entre ce même processus de régulation et la dépression d'autre part sont significatifs et témoignent de la présence de mécanismes spécifiques. En effet, on a pu observer que l'inhibition du partage social des émotions était liée au niveau d'anxiété, tandis que le niveau de dépression était davantage en lien avec l'inhibition d'une partie seulement du vécu émotionnel. On pourrait donc penser qu'il s'agirait ici de deux mécanismes spécifiques d'inhibition du partage social des émotions. Lorsque celui-ci est totalement inhibé («s'empêcher de partager son vécu émotionnel avec autrui »), on serait davantage dans un tableau clinique anxieux. De plus, dans des travaux antérieurs [20], nous avons pu montrer à

\footnotetext{
${ }^{1}$ Shame-Guilt Scale (Battacchi et collaborateurs, 1994) citée dans [30].
} 
un niveau descriptif que, lorsque le partage est inhibé c'est davantage pour des raisons personnelles (l'individu ne souhaite pas réactiver le vécu émotionnel négatif de l'événement). Par contre, lorsque le partage social n'est que partiellement inhibé (« expression incomplète du vécu émotionnel lors du partage avec autrui »), on serait en présence d'un tableau clinique teinté d'éléments dépressifs. Dans ce cas, il s'agirait d'une inhibition pour raisons sociales dans la mesure où l'individu n'exprime pas certains aspects estimés trop intimes et pouvant être mal jugés par autrui [20].

$\mathrm{Au}$ regard de ces considérations, on est amené à envisager le fonctionnement émotionnel spécifique à chaque symptomatologie à un niveau hypothétique pour l'instant. En effet, cette étude préliminaire pourra donner lieu à des analyses multivariées par la suite, une fois notre échantillon de sujets enrichi. De manière globale, dans l'anxiété, la tension forte ressentie physiologiquement au niveau symptomatique associée à une conscience émotionnelle de niveau bas entraînerait de façon complémentaire une diminution forte de la clarté cognitive. De ce fait, la régulation interne par les capacités d'identification et de représentation des émotions propres serait inefficace. Le processus de partage social des émotions, habituellement utilisé comme un moyen de régulation émotionnelle interpersonnelle, serait ici inhibé par les individus anxieux. En effet, inhiber ses émotions négatives permettrait de ne pas se confronter à ce qui rend ces individus anxieux (rappel de l'événement émotionnel) et à ce qui renforce leur anxiété (chercher à donner du sens à leur vécu subjectif). Concernant le fonctionnement émotionnel à l'œuvre dans la dépression, nous pensons qu'il s'agirait davantage d'une «hypersensibilité » ayant pour effet une focalisation sur le vécu émotionnel négatif venant perturber le processus de régulation interne (capacités de différenciation et d'identification des émotions). L'hypersensibilité porterait essentiellement sur le vécu émotionnel de l'individu dépressif mais également sur les émotions d'autrui. En effet, nous avons vu que la dépression était associée à des capacités de différenciation et d'identification des émotions de soi et d'autrui (relation positive avec le niveau de conscience émotionnelle inter-subjectif). Cependant, la conscience émotionnelle inter-subjective pourrait jouer un rôle dans l'expression complète du vécu émotionnel lors du partage social des émotions. Ceci aurait pour conséquence la mise en place d'un mécanisme d'inhibition d'une partie du vécu émotionnel chez ces individus. Il s'agirait de l'inhibition d'une partie du vécu émotionnel lors d'échanges socio-émotionnels afin de préserver le bienêtre d'autrui au détriment de son propre bien-être, en même temps que l'individu se préserve d'une image négative de soi. 
Ces résultats doivent néanmoins être considérés avec prudence, du fait de limites inhérentes, pour une part à la constitution de l'échantillon et pour une autre aux outils utilisés. Pour la première, notre échantillon étant relativement restreint et constitué en majeure partie de femmes, nous sommes conscients que la généralisation des résultats semble discutable. La présence notamment d'un sex-ratio plus équilibré permettrait sans doute de repérer des variations dues au sexe [8]. La question d'une dimension sociale sous-jacente à ce constat nécessiterait d'être approfondie. Une autre limite réside dans l'utilisation d'outils psychométriques. Même si ils ont été choisis en fonction de leurs caractéristiques métrologiques supposées acceptables, il n'en reste pas moins qu'ils sont soumis à l'influence de multiples facteurs inhérents à l'auto-évaluation. Il serait nécessaire de poursuivre nos investigations en croisant une méthodologie qualitative à celle quantitative afin de mieux structurer le protocole et tenter d'écarter les redondances et biais de réponse. Une dernière limite à notre étude réside dans la réalisation d'une évaluation uniquement transversale alors que la plupart des questions posées sur les liens entre les éléments du fonctionnement émotionnel des sujets et leurs symptômes nécessiteraient une approche longitudinale. Cependant, nous avons fait le choix dans cette étude exploratoire des relations entre conscience émotionnelle, partage social des émotions et anxiété-dépression de nous intéresser à l'état actuel des sujets, hors du champ de la causalité psychique.

Enfin, nos résultats soulèvent des pistes significatives de réflexion quant à la prise en charge des états anxieux et dépressifs. En effet, nous pensons qu'il s'agirait avant tout de travailler davantage en lien avec les médecins généralistes qui constituent les interlocuteurs privilégiés des souffrances du quotidien. Ces derniers ne sont pas toujours au fait des différentes techniques, approches et lieux de prise en charge thérapeutique pouvant exister en psychologie. Ceci permettrait sans doute de faciliter le travail d'orientation des patients vers une prise en charge psychologique, comme il est conseillé en cas de traitement chimique [28]. D'un point de vue clinique, il paraît tout à fait essentiel d'appréhender les états anxieux et dépressifs de façon distincte et de prendre en compte la dimension sociale qui semble jouer un rôle important dans la verbalisation des émotions. 


\section{Références}

[1] Bagby, R.M., Taylor, G.J., Ryan, D. Toronto Alexithymia Scale : Relationship with personality and psychopathology measures.Psychotherapy and Psychosomatics 1986, 45 : 207-215.

[2] Barczack, P., Kane, N., Andrews, S., et al. Patterns of psychiatric morbidity in a genitourinary clinic: a validation of the Hospital Anxiety Depression scale (HAD). Brit. J. Psychiatry 1988, $152:$ 698-700.

[3] Berthoz, S., Consoli, S., Perez-Diaz, F., et al.Alexithymia and anxiety : compounded relationships? A psychometric study. Eur Psychiatry 1999, 14 : 372-378.

[4] Berthoz, S., Ouhayoun, B., Parage, N., et al. Etude préliminaire des niveaux de conscience émotionnelle chez des patients déprimés et des contrôles. Ann Med-psychol 2000, $158(8)$ : 665-672.

[5] Butler, E.A. Emotional conversations: regulation of emotions. Dissertation Abstracts International Section B. The Sciences \& Engineering 2004, 65(4-B) : 2082.

[6] Bydlowski, S., Corcos, M., Paterniti, S., et al. Validation de la version française de l'échelle des niveaux de conscience émotionnelle. L'Encéphale 2002, 28(4), 310-320.

[7] Ciarrochi, J., Scott, G. The link between emotional competence and well-being: a longitudinal study. Br J of Guid and Consel 2006, (34) 2: 231-43.

[8] Hauwel-Fantini, C., Pedinielli, J.-L. De la non-expression à la surexpression des émotions ou comment l'expérience émotionnelle repose la question des liens entre sexe, alexithymie et répression. Ann Med-Psychol 2008, $166: 277-284$.

[9] Hintikka, J., Honkalampi, K., Lehtonen, J., et al. Are alexithymia and depression distinct or overlapping construct ? : A study in a general population. Compr Psychiatry 2001, 42 : 234-9.

[10] Honkalampi K., Hintikka, J., Tanskanen, et al. Depression is strongly associated with alexithymia in the general population.Journal of Psychosomatic Research 2000, 48:99104.

[11] Jouvent, R., Bungener, C., Morand, P., et al. M. Distinction trait/état et anxiété en médecine générale - Etude descriptive . L'Encéphale 1999, XXV (1) : 44-49

[12] Kovess, V., Chastang, F. Les déprimés et le système de soins. Inf. Psychiatr. 1998, 74(4): 363-71.

[13] Lane, RD., Quinlan, D.M., Schwartz, GE., et al. The levels of emotional awareness scale: a cognitive-developmental measure of emotion. J of Pers Asses 1990, 55(1\&2), 124134. 
[14] Lane, R.D., Schwartz, G.E. Levels of emotional awareness: a cognitve-developmental theory and its application to psychopathology. Amer J of Psych 1987, 144: 133-43.

[15] Lane, R.D., Sechrest, L., Riedel, R., et al. Pervasive emotion recognition deficit common to alexithymia and the repressive coping style. Psychosom Med 2000, 62: 492-501.

[16] Lépine, J.P., Godchau, M., Brun, P., et al. Evaluation de l'anxiété et de la dépression chez des patients hospitalisés dans un service de médecine interne. Ann Med-psychol $1985,143: 175-189$.

[17] Marchesi, C., Brusamonti, E., Maggini, C. Are alexithymia, depression and anxiety distinct constructs in affective disorders? J of Psychosom Res 2000, $49: 43-49$.

[18] Nandrino, J.L. Comprendre et accompagner la personne dépressive. Levallois Perret, Gaëtan Morin ; 1999.

[19] Parquet, P.J., Chevallier, L., Cuche, H., et al.Itinéraires des déprimés. Réflexions sur leurs trajectoires en France. Rapport d'experts, 2001.

[20] Pasquier, A., Bonnet, A., Pedinielli, J.L. Anxiété, dépression et partage social des émotions : Des stratégies de régulation émotionnelle spécifiques. Journal des Thérapies Cognitive et Comportementale 2008, $18: 2-7$.

[21] Pasquier, A., Bonnet, A., Pedinielli, J.L. Fonctionnement cognitivo-émotionnel : le rôle de l'intensité émotionnelle chez les individus anxieux. Ann Med Psychol, sous presse.

[22] Pedinielli, J.L. Psychosomatique et alexithymie. Paris, PUF ; 1992.

[23] Pelissolo, A. Anxiété, dépression : Des troubles anxieux à la dépression. Paris, Phase 5 ; 2006.

[24] Power, M.J., Tarsia, M. Basic and Complex Emotions in Depression and Anxiety. Clin. Psychol. Psychother. 2007, 14 : 19-31.

[25] Rimé, B. Le partage social des émotions. In B. Rimé \& K. Scherer (Eds.). Les émotions (pp. 271-303). Neuchâtel, Delachaux \& Niestlé ; 1989.

[26] Rimé, B. Le partage social des émotions. Paris, Presses Universitaires de France ; 2005.

[27] Rimé, B. Interpersonal emotion regulation. In J.J. Gross (Ed.), Handbook of emotion regulation (pp. 466-485). New York, The Guilford Press ; 2007.

[28] Rouillon, F., Lejoyeux, M., Le Gep, F. Modalités d'accès aux soins en consultation psychiatrique, Ann Méd Psychol1993,151(9) : 628-632.

[29] Sifnéos, P.E. The prevalence of alexithymic characteristics in psychosomatic patients. Psychother Psychosom 1973, 22 : 255-62. 
[30] Suslow, T., Donges, U.-S., Kersting, A., et al. 20-Item Toronto Alexithymia Scale: Do difficulties describing feelings assess proneness to shame instead of difficulties symbolizing emotions?, Scand J of Psychol 2000, 41(3) : 329-334.

[31] Taylor, G.J., Bagby, R.M., Parker, J.D.A. Disorders of affect regulation. Alexithymia in medical and psychiatric illness. Cambridge, Cambridge and Univerversity Press ; 1997.

[32] Zigmond, A.S., Snaith, R.P.The Hospital Anxiety and Depression Scale. Acta Psychiatr Scand 1983, 67: 361-370. 


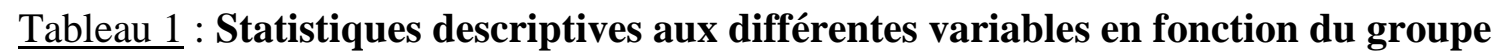

\begin{tabular}{|l|c|c|c|c|}
\hline & Groupe & Moyenne & Ecart-type & N \\
\hline Anxiété & $\mathrm{AD}$ & $12,28^{* * *}$ & 2,65 & 60 \\
& $\mathrm{NAD}$ & 5,51 & 1,28 & 47 \\
\hline Dépression & $\mathrm{AD}$ & $4,90^{* * *}$ & 3,32 & 60 \\
& $\mathrm{NAD}$ & 2,64 & 1,48 & 47 \\
\hline NCE-Globale & $\mathrm{AD}$ & $55,18^{* *}$ & 7,76 & 60 \\
& $\mathrm{NAD}$ & 60,11 & 7,85 & 47 \\
\hline NCE-Intra & $\mathrm{AD}$ & $48,05^{* * *}$ & 8,90 & 60 \\
\hline NCE-Inter & $\mathrm{NAD}$ & 54,02 & 7,20 & 47 \\
& $\mathrm{AD}$ & $46,02^{*}$ & 7,82 & 60 \\
\hline PSE : inhibition & $\mathrm{NAD}$ & 48,96 & 6,33 & 47 \\
totale & $\mathrm{AD}$ & 0,42 & 1,21 & 47 \\
\hline PSE : inhibition & $\mathrm{NAD}$ & 0,30 & 0,46 & 60 \\
partielle & $\mathrm{AD}$ & 7,35 & 3,05 & 47 \\
\hline
\end{tabular}

*** $\mathrm{t}$ de Student significatif à $\mathrm{p}<.0001 ; * * \mathrm{t}$ significatif à $\mathrm{p}=.001 ; * \mathrm{t}$ significatif à $\mathrm{p}=.03$

Tableau 2 : Corrélations entre états anxieux, états dépressifs et conscience émotionnelle

\begin{tabular}{|ll|c|c|c|c|}
\hline & & Anxiété & NCE-Globale & NCE-Intra & NCE-Inter \\
\hline Anxiété & $\mathbf{r}$ & - & -.26 & -.27 & -.26 \\
& $\mathbf{p}$ & & .04 & .03 & .04 \\
\hline Dépression & $\mathbf{r}$ & .34 & .37 & .29 & .28 \\
& $\mathbf{p}$ & .007 & .003 & .02 & .03 \\
\hline
\end{tabular}

(NCE-G : Niveau de Conscience Emotionnelle Global/ NCE-Intra : Niveau de Conscience Emotionnelle Intrasubjectif/ NCE-Inter : Niveau de Conscience émotionnelle Inter-subjectif) 